\title{
Superior Vena Cava
}

National Cancer Institute

\section{Source}

National Cancer Institute. Superior Vena Cava. NCI Thesaurus. Code C12816.

The large vein that terminates in the right atrium and transports deoxygenated blood from the head, neck, arms, and chest to the heart. 